\section{Recurrencia de la estenosis de uretra bulbar en pacientes con tratamiento primario de uretroplastia anastomósica o uretrotomía interna}

Trujillo-Ortiz Luis, ${ }^{1}$ Morales-Montor $\mathrm{G},{ }^{2}$ Cantellano-Orozco $\mathrm{M},{ }^{2}$ MartínezArroyo C, ${ }^{2}$ Fernández-Noyola G, ${ }^{2}$ Sedano-Basilio J, ${ }^{1}$ Pacheco-Gahbler $\mathrm{C}^{3}$

Resumen

ANTECEDENTES: la estenosis uretral reduce el flujo de orina y dificulta el vaciado vesical; su origen es multifactorial y no existe un consenso definitivo respecto de cómo se establecen el diagnóstico y el tratamiento definitivo.

OBJETIVO: determinar la recurrencia de estenosis de uretra bulbar en pacientes con intervención quirúrgica primaria de uretroplastia anastomósica o uretrotomía interna.

MATERIALES Y MÉTODOS: estudio de cohorte, retrospectivo, efectuado en pacientes a quienes se practicó uretroplastia anastomósica y uretrotomía interna, entre 2007 y 2014, en el Hospital General Dr. Manuel Gea González, Ciudad de México. Análisis de características preoperatorias: edad, retención urinaria, causa, longitud y localización del segmento estenósico; resultados pre y posquirúrgico de la uroflujometría $\left(\mathrm{Q}_{\max }\right)$, recurrencia de la estenosis y tratamiento posterior a ésta.

RESULTADOS: se registraron 31 (22.3\%) pacientes a quienes se efectuó uretroplastia anastomósica y 108 uretrotomía interna (77.7\%); la edad media fue de $54.12 \pm 14.6$ años y la causa más frecuente fue iatrogénica $(57.6 \%)$, seguida del traumatismo (29.5\%), idiopática $(8.6 \%)$ e infecciosa $(4.3 \%)$. La longitud media de la estenosis fue de $13.20 \pm 4.9 \mathrm{~mm}$; retención urinaria en $5.8 \%$; $\mathrm{Q}_{\max }$ prequirúrgico de $6.81 \pm 1.53$ y posquirúrgico de $11.78 \pm 2.44 \mathrm{~mL} / \mathrm{seg}$. No se observó recurrencia a seis meses en los pacientes tratados mediante uretroplastia anastomósica y la recurrencia en los de uretrotomía interna fue de $43.9 \%$. En los pacientes con recurrencia a 6 meses, el $Q_{\max }$ posquirúrgico fue $10.77 \pm 1.82$ vs $12.5 \pm 2.59 \mathrm{~mL} / \mathrm{seg}$ de quienes no tuvieron recurrencia $(p=0.002)$.

CONCLUSIONES: la uretroplastia anastomósica puede considerarse el tratamiento de primera línea en pacientes con estenosis de uretra bulbar $\leq 2 \mathrm{~cm}$ de longitud. La uretrotomía interna representa la técnica quirúrgica de mínima invasión de mayor disponibilidad para la práctica urológica.

PALABRAS CLAVE: estenosis de uretra, estrechez uretral, uretroplastia anastomósica, uretrotomía interna.

\footnotetext{
${ }^{1}$ Residente del servicio de Urología.

${ }^{2}$ Médico adscrito al servicio de Urología.

${ }^{3}$ Jefe del servicio de Urología.
}

Hospital General Dr. Manuel Gea González, Ciudad de México.

Recibido: mayo 2017

Aceptado: mayo 2017

Correspondencia

Luis Trujillo Ortiz

drtrujillo68@gmail.com

Este artículo debe citarse como

Trujillo-Ortiz L, Morales-Montor G, CantellanoOrozco M, Martínez-Arroyo C, Fernández-Noyola G, Sedano-Basilio J, Pacheco-Gahbler C. Recurrencia de la estenosis de uretra bulbar en pacientes con tratamiento primario de uretroplastia anastomósica o uretrotomía interna. Rev Mex Urol. 2017 mayo;77(3):191-198. 


\section{Recurrence of bullbar urethral stricture in patients with primary treatment of anastomotic urethroplasty or internal urethrotomy}

Trujillo-Ortiz Luis, ${ }^{1}$ Morales-Montor $\mathrm{G}^{2}$ Cantellano-Orozco $\mathrm{M},{ }^{2}$ MartínezArroyo C, ${ }^{2}$ Fernández-Noyola G, ${ }^{2}$ Sedano-Basilio J, ${ }^{1}$ Pacheco-Gahbler $\mathrm{C}^{3}$

\begin{abstract}
BACKGROUND: Urethral stricture is an alteration that reduces urine flow and hampers bladder voiding. Its origin is multifactorial and there is no definitive consensus on approach or standard treatment.
\end{abstract}

OBJECTIVE: To determine the recurrence of bulbar urethral stricture in patients that underwent primary anastomotic urethroplasty or primary internal urethrotomy.

MATERIALES AND METHODS: A retrospective cohort study was conducted on patients that underwent anastomotic urethroplasty or internal urethrotomy within the time frame of 2007 and 2014 at the Hospital General Dr. Manuel Gea González, in Mexico City. The preoperative characteristics analyzed were: age, urinary retention, etiology, length and location of the strictured segment, preoperative and postoperative uroflowmetry (Qmax) results, stricture recurrence, and treatment after recurrence.

RESULTS: Thirty-one (22.3\%) patients that underwent anastomotic urethroplasty and $108(77.7 \%)$ that had internal urethrotomy were registered. Mean patient age was $54.12 \pm 14.6$ years and the most frequent etiology was iatrogenic $(57.6 \%)$, followed by traumatic causes $(29.5 \%)$, idiopathic causes (8.6\%), and infectious origin (4.3\%). Mean stricture length was $13.20 \pm 4.9 \mathrm{~mm}, 5.8 \%$ of patients had urinary retention, preoperative Qmax was $6.81 \pm 1.53$, and postoperative Qmax was $11.78 \pm 2.44 \mathrm{~mL} / \mathrm{sec}$. There was no recurrence at 6 months in the patients that underwent anastomotic urethroplasty. Recurrence in the patients that had internal urethrotomy was $43.9 \%$. Postoperative Qmax was $10.77 \pm 1.82 \mathrm{~mL} / \mathrm{sec}$ in the patients that had recurrence at 6 months $v s 12.5 \pm 2.59 \mathrm{~mL} / \mathrm{sec}$ in those that did not have recurrence $(p=0.002)$.

CONCLUSIONS: Anastomotic urethroplasty can be considered firstline treatment in patients with bulbar urethral stricture $\leq 2 \mathrm{~cm}$ in length. Internal urethrotomy is the minimally invasive surgical treatment of greater availability in urologic practice.

KEY WORDS: Urethral stricture, urethral narrowness, anastomotic urethroplasty, internal urethrotomy

\footnotetext{
${ }^{1}$ Residente del servicio de Urología. ${ }^{2}$ Médico adscrito al servicio de Urología.

${ }^{3}$ Jefe del servicio de Urología.

Hospital General Dr. Manuel Gea González, Ciudad de México.

Correspondence Luis Trujillo Ortiz drtrujillo68@gmail.com
} 


\section{ANTECEDENTES}

La estenosis uretral, como su nombre indica, es el estrechamiento de la uretra, provocado por espongiofibrosis isquémica y que se manifiesta como tejido cicatricial en la porción del cuerpo esponjoso. La contracción de este tejido de cicatriz disminuye el calibre de la uretra y, a su vez, el flujo de la orina, y dificulta el vaciado vesical. ${ }^{1}$

La estenosis uretral es el término más común para referirse a la disminución del calibre de un segmento de la uretra rodeado por el cuerpo esponjoso; es decir, desde el meato uretral hasta la uretra bulbar. El término estenosis de uretra se utiliza para describir la disminución del calibre en la porción comprendida entre la uretra prostática y el cuello vesical. La Organización Mundial de la Salud y la Sociedad Internacional de Urología, en la Conferencia Internacional de Trastornos urológicos (ICUD), sugirieron describir a la uretra en términos anatómicos específicos, en lugar de segmentos anterior y posterior. $^{2}$

La prevalencia de estenosis uretral en Estados Unidos se estima entre 200 por cada 100,000 hombres jóvenes y más de 600 por cada 100,000 hombres mayores de 65 años de edad. En ese mismo país, los datos del Instituto de Salud estimaron una incidencia de $0.9 \%$ en 2001 , aunque su cifra real se desconoce. ${ }^{3}$

La incidencia de estenosis (estrechez) de uretra en la población mexicana se desconocce; sin embargo, tiene repercusión negativa en quienes la padecen y se ha llegado a calcular un gasto anual en el Sector Salud de 200 millones de pesos. $^{4}$

La estenosis de uretra puede originarse por múltiples causas, por ejemplo: anomalías congénitas, infecciones, traumatismo perineal, instrumentación uretral, cateterismo vesical, reparación errónea de hipospadias y enfermedades inflamatorias del cuerpo esponjoso (principalmente las causadas por liquen escleroso o balanitis xerótica obstructiva). ${ }^{5}$

Las causas inflamatorias y traumáticas representan entre 15 y $19 \%$ de las estenosis, respectivamente. ${ }^{6}$ El traumatismo sigue siendo la causa más frecuente en los países en vías de industrialización (36\% secundarias a traumatismos y $95 \%$ de las lesiones como causas relacionadas). ${ }^{7}$

Las estenosis idiopáticas suelen originarse en la uretra bulbar y afectan, con mayor frecuencia, a los varones jóvenes que a los de mayor edad (48 vs 23\%, respectivamente). En estos últimos, la causa más común se asocia con cirugía transuretral o colocación de sondas uretrales permanentes a largo plazo. La estenosis de la uretra posterior se produce en $5-10 \%$ de los pacientes y resulta de la cirugía de próstata o intervención para el cáncer de próstata. ${ }^{8}$

En cuanto a la evaluación clínica, no existe un consenso definitivo respecto del mejor protocolo para el diagnóstico y tratamiento de la estenosis uretral. Para decidir el tratamiento se requiere determinar: localización y longitud de la obstrucción, y la asociación de la patología uretral. ${ }^{9}$

El estudio recomendado para la evaluación y localización de la estenosis uretral es la uretrografía retrógrada, cuya sensibilidad varía de 75 a $100 \%$ y la especificidad de 72 a $97 \%$. La cistoscopia se considera la técnica de elección para diagnosticar la estenosis uretral. El cistouretrograma es un estudio complementario para evaluar el cuello de la vejiga y la uretra posterior, especialmente en sujetos con estenosis uretral posterior. La ecografía uretral tiene mayor sensibilidad en la evaluación de la longitud de la estenosis, el diámetro y grado de la espongiofibrosis que el cistouretrograma; sin embargo, 
representa un estudio de complemento y se requiere personal adiestrado para su ejecución. ${ }^{9,10}$

La uroflujometría es una de las técnicas básicas de investigación clínica en la práctica urológica diaria. Este procedimiento correlaciona el volumen, tiempo y velocidad en el que la vejiga expulsa la orina a través de la uretra, permitiendo al urólogo valorar clínicamente si existe un patrón miccional fisiológico o anormal. Es un método no invasivo y relativamente económico; por lo tanto, se considera el estudio de primera línea en cualquier paciente con sospecha de disfunción de la vía urinaria inferior. El flujo máximo urinario (Qmax) es una variable estimada mediante uroflujometría, pero no es lo suficientemente sensible para sustituir a la cistoscopia y establecer el diagnóstico de estenosis, sobre todo en pacientes jóvenes, donde las tasas de flujo de base son superiores; no obstante, forma parte de los estudios complementarios de recurrencia de estenosis en todos los pacientes. ${ }^{11,12}$

Entre las principales opciones de tratamiento se encuentran: dilatación uretral, uretrotomía interna y uretroplastia (anastomósica y de aumento). El objetivo de la dilatación uretral es alargar la cicatriz sin desgarrar la mucosa, lo que permite la ampliación gradual en la luz uretral; sin embargo, no existe una clara recomendación de su práctica. ${ }^{13}$

La uretrotomía interna (incisión de la estenosis uretral con visión directa) sigue siendo el tratamiento de elección, pues un estudio reciente reveló que $82.5 \%$ de los urólogos certificados en Estados Unidos pone en práctica este método en pacientes con estenosis uretral. La mayor parte de la bibliografía que apoya el tratamiento de la uretrotomía interna se compone de evidencia nivel 3 (revisión de series), donde evalúan los resultados a corto plazo y reportan tasas de éxito variables, incluso de 22 a $100 \% .^{14}$
La uretroplastia se considera el patrón de referencia del tratamiento de la estenosis uretral. Los datos actuales para uretroplastia anastomósica (uretroplastia de escisión y anastomosis) y aplicación de injertos (uretroplastia de aumento) señalan mayores tasas de éxito a largo plazo que el resto de las opciones de tratamiento. La relación costo-efectividad del tratamiento de la estenosis uretral sugiere que la uretroplastia inmediata o un solo intento de uretrotomía interna es más rentable que la dilatación a largo plazo.

La uretroplastia anastomósica (escisión y anastomosis primaria) es la escisión de la cicatriz y la reconexión de la uretra. Esta técnica se considera un tratamiento efectivo para la estenosis uretral bulbar, independientemente de la causa o tratamiento previo. Se indica en pacientes con estenosis igual o menor de $2 \mathrm{~cm}$ (incluso en quienes tienen estenosis de mayor longitud), con excelente tasa de éxito (90-95\%) a largo plazo. En general, la tasa de complicaciones de la uretroplastia anastomósica es baja (menos de $10 \%$ ) y la mayoría de los pacientes se curan entre los 6 y 12 meses. $^{15}$

El objetivo de este estudio consiste en determinar la recurrencia de estenosis de uretra bulbar en pacientes con intervención quirúrgica primaria de uretroplastia anastomósica o uretrotomía interna, además de describir las características pre y posquirúrgicas, causa, flujo urinario máximo, tiempo de manifestación y tratamiento de la recurrencia.

\section{MATERIALES Y MÉTODOS}

Estudio observacional, descriptivo, retrospectivo y transversal, en el que se evaluaron los expedientes de pacientes iguales o mayores de 14 años de edad, con diagnóstico de estenosis de uretra bulbar, que recibieron tratamiento primario con uretrotomía interna o uretroplas- 
tia anastomósica en el servicio de Urología del Hospital General Dr. Manuel Gea González, entre 2007 y 2014.

El número de sujetos se definió por conveniencia. Los datos se obtuvieron de los reportes intraoperatorios realizados en el servicio de Urología y de los expedientes del archivo clínico del Hospital General Dr. Manuel Gea González.

Se analizaron características preoperatorias como: edad, retención urinaria, causa, longitud y localización del segmento estenósico; resultados pre y posquirúrgicos de la uroflujometría (Qmax), recurrencia y tratamiento implementado después de ésta.

Para el análisis estadístico se utilizaron pruebas de tendencia central y porcentajes, además de $\chi^{2}$ para medidas de asociación en variables cualitativas. Se consideró significativamente estadístico el valor de $\mathrm{p}<0.05$.

\section{RESULTADOS}

Se encontraron 139 pacientes con diagnóstico de estenosis de uretra bulbar $(\leq 2): 31(22.3 \%)$ a quienes se practicó uretroplastia anastomósica y a $108(77.7 \%)$ uretrotomía interna. La media de edad fue de $54.12 \pm 14.6$ años (límites de 18-88). La causa más frecuente fue la iatrogenia en $80(57.6 \%)$ casos, seguida del traumatismo en $41(29.5 \%)$, causas idiopáticas en 12 (8.6\%) e infecciosas en 6 (4.3\%). La longitud media de la estenosis fue de $13.20 \pm 4.9 \mathrm{~mm}(5-20 \mathrm{~mm})$; con retención urinaria en $8(5.8 \%)$ casos. El $\mathrm{Q}_{\max }$ prequirúrgico medio fue de $6.81 \pm 1.53(3-11$ $\mathrm{mL} / \mathrm{seg}$ ) y el posquirúrgico de $11.78 \pm 2.44 \mathrm{~mL} /$ seg (5-17 mL/seg). No se observó recurrencia a seis meses en los pacientes tratados mediante uretroplastia anastomósica; sin embargo, ésta apareció en $43.9 \%$ de quienes tuvieron uretrotomía interna (Cuadro 1).
Cuadro 1. Pacientes con estenosis de uretra bulbar $\leq 2 \mathrm{~cm}$

\begin{tabular}{|c|c|c|}
\hline Variable & $\mathbf{n}$ & $\%$ \\
\hline $\begin{array}{l}\text { Edad (años) } \\
\text { Media } \pm \text { DE } \\
\text { Límites }\end{array}$ & $\begin{array}{c}54.12 \pm 14.6 \\
18-88\end{array}$ & \\
\hline Pacientes (n) & 139 & \\
\hline $\begin{array}{l}\text { Etiología (n) } \\
\text { latrogenia } \\
\text { Traumatismo } \\
\text { Idiopática } \\
\text { Infecciosa }\end{array}$ & $\begin{array}{c}80 \\
41 \\
12 \\
6\end{array}$ & $\begin{array}{c}57.6 \\
29.5 \\
8.6 \\
4.3\end{array}$ \\
\hline $\begin{array}{l}\text { Longitud }(\mathbf{m m}) \\
\text { Media } \pm \mathrm{DE} \\
\text { Límites }\end{array}$ & $\begin{array}{c}13.2 \pm 4.92 \\
5-20\end{array}$ & \\
\hline $\begin{array}{l}\text { Qmax prequirúrgico }(\mathbf{m L} / \mathbf{s e g}) \\
\text { Media } \pm \mathrm{DE} \\
\text { Límites }\end{array}$ & $\begin{array}{c}6.81 \pm 1.53 \\
3-11\end{array}$ & \\
\hline $\begin{array}{l}\text { Qmax posquirúrgico }(\mathbf{m L} / \mathbf{s e g}) \\
\text { Media } \pm \mathrm{DE} \\
\text { Límites }\end{array}$ & $\begin{array}{c}11.78 \pm 2.44 \\
5-17\end{array}$ & \\
\hline $\begin{array}{l}\text { Tipo de cirugía (n) } \\
\text { UIVD } \\
\text { Uretroplastia }\end{array}$ & $\begin{array}{c}108 \\
31\end{array}$ & $\begin{array}{l}77.7 \\
22.3\end{array}$ \\
\hline $\begin{array}{l}\text { Recurrencia a } 6 \text { meses (n) } \\
\text { UIVD } \\
\text { Uretroplastia }\end{array}$ & $\begin{array}{c}61 \\
0\end{array}$ & $\begin{array}{c}43.9 \\
0\end{array}$ \\
\hline $\begin{array}{l}\text { Reintervención* (n) } \\
\text { Uretroplastia } \\
\text { UIVD } \\
\text { Otra }\end{array}$ & $\begin{array}{c}0 \\
25 \\
36\end{array}$ & $\begin{array}{c}0 \\
18 \\
25.9\end{array}$ \\
\hline
\end{tabular}

*Cirugía efectuada para el tratamiento de la recurrencia; DE: desviación estándar; Qmax: flujo urinario máximo; UIVD: uretrotomía interna visual directa.

En el subanálisis comparativo de los pacientes con recurrencia en los primeros seis meses se encontró significación estadística al comparar el Qmax posquirúrgico $(\mathrm{p}=0.002)$ y el tipo de cirugía efectuada como tratamiento primario de estenosis uretral $(p<0.001)$. Cuadro 2.

\section{DISCUSIÓN}

Los pacientes con estenosis de uretra bulbar igual o menor de $2 \mathrm{~cm}$ de longitud pueden tratarse mediante uretroplastia anastomósica o uretrotomía interna. Como su nombre lo in- 
Cuadro 2. Características de los pacientes con estenosis bulbar $\leq 2 \mathrm{~cm}$

\begin{tabular}{|c|c|c|c|}
\hline Variable & $\begin{array}{l}\text { Sin } \\
\text { recurrencia }\end{array}$ & $\begin{array}{c}\text { Con } \\
\text { recurrencia }\end{array}$ & $\mathbf{p}$ \\
\hline Pacientes n (\%) & $78(52.3 \%)$ & $61(56.3 \%)$ & \\
\hline $\begin{array}{l}\text { Edad (años) } \\
\text { Media } \pm \text { DE }\end{array}$ & $52.33 \pm 12.6$ & $56.39 \pm 16.7$ & \\
\hline $\begin{array}{l}\text { Etiología (\%) } \\
\text { latrogenia } \\
\text { Traumatismo } \\
\text { Idiopática } \\
\text { Infecciosa }\end{array}$ & $\begin{array}{c}49(61.3 \%) \\
17(41.5 \%) \\
10(83.3 \%) \\
2(33.3 \%)\end{array}$ & $\begin{array}{c}31(38.8 \%) \\
24(58.5 \%) \\
2(16.7 \%) \\
4(66.7 \%)\end{array}$ & \\
\hline $\begin{array}{l}\text { Longitud }(\mathbf{m m}) \\
\text { Media } \pm \text { DE }\end{array}$ & $12.50 \pm 5.1$ & $14.10 \pm 4.5$ & 0.053 \\
\hline $\begin{array}{l}\text { Qmax } \\
\text { prequirúrgico } \\
(\mathbf{m L} / \mathbf{s e g}) \\
\quad \text { Media } \pm \text { DE }\end{array}$ & $7.08 \pm 1.65$ & $6.48 \pm 1.29$ & 0.195 \\
\hline $\begin{array}{l}\text { Qmax } \\
\text { posquirúrgico } \\
(\mathbf{m L} / \mathbf{s e g}) \\
\quad \text { Media } \pm \text { DE }\end{array}$ & $12.5 \pm 2.59$ & $10.77 \pm 1.82$ & 0.002 \\
\hline $\begin{array}{l}\text { Tipo de } \\
\text { cirugía (n) } \\
\text { Uretroplastia } \\
\text { UIVD }\end{array}$ & $\begin{array}{l}31(100 \%) \\
47(43.5 \%)\end{array}$ & $61 \stackrel{-}{(56.5)}$ & $\begin{array}{l}<0.001 \\
<0.001\end{array}$ \\
\hline $\begin{array}{l}\text { Reintervención* } \\
\text { (n) } \\
\text { UIVD } \\
\text { Otro } \\
\text { (no identificado) }\end{array}$ & $\begin{array}{l}- \\
-\end{array}$ & $\begin{array}{c}25(18 \%) \\
36(25.9 \%)\end{array}$ & \\
\hline
\end{tabular}

*Cirugía efectuada para el tratamiento de la recurrencia; DE: desviación estándar; Qmax: flujo urinario máximo; UIVD: uretrotomía interna visual directa.

dica, la uretroplastia anastomósica (escisión y anastomosis) implica la transección del cuerpo esponjoso y la escisión de la estenosis uretral, con la movilización distal y proximal de la uretra antes de realizar una anastomosis libre de tensión. Terlecki y su grupo reportaron que la uretroplastia anastomósica es efectiva en pacientes con estenosis bulbar proximal de $5 \mathrm{~cm}$; $\sin$ embargo, suele limitarse en sujetos con estenosis igual o menor de $3 \mathrm{~cm}$. Un estudio que evaluó 210 uretroplastias (2007-2009) señaló 112 casos con estenosis de uretra bulbar y de éstos a 72
(64\%) se les realizó uretroplastia anastomósica. La longitud media fue de $2 \mathrm{~cm}(1$ a 5 ) y 31 de 72 casos de estenosis (43\%) tuvieron longitud intermedia (2.5 a $5 \mathrm{~cm})$. Solo se observó recurrencia en $1(1.4 \%)$ paciente. $^{16}$

La uretroplastia anastomósica es una opción fácilmente reproducible y puede evitar complejidades técnicas, además de morbilidad por colocación de injertos, colgajos o ambos. ${ }^{17,18}$ Un metanálisis de 17 series, con 1,234 casos, confirmó $93 \%$ de éxito de la uretroplastia anastomósica en el tratamiento de la estenosis de uretra bulbar, con mínimas y transitorias complicaciones. ${ }^{18}$

Granieri y sus colaboradores (2014) revisaron, de forma retrospectiva, 384 pacientes a quienes se efectuó uretroplastia (1996-2011), con promedio de edad de 43.2 años (límites de 15-85) y longitud media de la estenosis de $2 \mathrm{~cm}(0.2-$ 10). La causa más frecuente registrada fue la idiopática en 233 (54.3\%) casos. La uretroplastia anastomósica fue la técnica reconstructiva más practicada, en 202 (52.6\%) pacientes. Durante el periodo de estudio la longitud media de la estenosis de pacientes tratados con uretroplastia anastomósica fue de $1.4 \mathrm{~cm}(0.2-4.5)$ y el seguimiento medio de 16.8 meses. En general, $26(7 \%)$ pacientes tuvieron recurrencia a 11.2 meses después de la operación. El tratamiento posterior a la recurrencia consistió en dilatación endoscópica o uretrotomía interna. ${ }^{19}$

Fall y sus coautores (2014), en su estudio prospectivo y monocéntrico (2007-2010) de uretroplastias (anastomósica y de aumento) con 75 pacientes, evaluaron variables como: edad, circunstancias del diagnóstico, localización y longitud de la estenosis; causa y procedimientos uretrales anteriores; experiencia del personal en cirugía reconstructiva uretral y tipo de uretroplastia. Los resultados obtenidos mostraron que en $60 \%$ de los casos la causa más común 
fue la uretritis por infecciones de trasmisión sexual. La edad media de los pacientes fue de $48.3 \pm 20$ años (8-87 años), con mediana de 50 años. El síntoma predominante fue la retención urinaria $(62.7 \%)$. La principal localización fue en la uretra bulbar (63.3\%) y el promedio de longitud de la estenosis de $2.45 \pm 1.39 \mathrm{~cm} \mathrm{(1-7}$ $\mathrm{cm}$ ), con mediana de $2 \mathrm{~cm}$. La longitud promedio de las estenosis tratadas mediante uretroplastia anastomósica fue de $1.95 \pm 0.72 \mathrm{~cm}$ (media de $2 \mathrm{~cm}$ ). La tasa de éxito de la uretroplastia fue de $92.3 \%$ para estenosis de $1 \mathrm{~cm}$ de largo y de $64.6 \%$ para las de 1 a $3 \mathrm{~cm}$. El resultado de la uretroplastia no dependió, significativamente, de la ubicación de la estenosis. En el estudio aquí realizado, la uretroplastia anastomósica mostró mejores resultados que el resto de las técnicas. ${ }^{20}$

En México, Cisneros y su grupo (2013) efectuaron un estudio retrospectivo en 80 (de 2005 a 2012) pacientes tratados por estenosis en el Centro Médico ISSEMYM, de los que 59 se trataron, de manera endoscópica, mediante uretrotomía interna y 19 uretroplastia (un paciente fue objeto de ambos procedimientos). La edad promedio de los pacientes fue de 51 años (22-80 años) y la causa más frecuente fue la iatrogenia. La uretra bulbar fue la porción más afectada en $51.2 \%$ $(\mathrm{n}=40)$. En los pacientes con uretrotomía interna se registró una longitud media de la estenosis de $1.2 \mathrm{~cm}(0.8-1.6 \mathrm{~cm}) ; 33$ (55.93\%) tuvieron recurrencia en el primer semestre del seguimiento. En los pacientes tratados mediante uretroplastia se encontró una longitud media de $2.20 \mathrm{~cm}(0.5-4$ $\mathrm{cm}$ ) y la tasa de éxito fue de $94.7 \%{ }^{21}$

En el tratamiento primario de la estenosis de uretra, aun teniendo como referente la evidencia previa, la uretrotomía interna sigue siendo el método más utilizado para la estenosis de uretra anterior. La simplicidad y rapidez del tratamiento endoscópico en pacientes ambulatorios ha contribuido con su popularidad; sin embargo, los datos de eficacia a largo plazo han sido insuficientes. Los mejores resultados de la uretrotomía interna se reportan en pacientes con estenosis cortas y no traumáticas de la uretra bulbar, pues se han asociado con una tasa de éxito de $39-73 \%{ }^{22,23}$ Aunque la uretrotomía interna sugiere una tasa de éxito menor, la mayoría de los urólogos realiza procedimientos endoscópicos repetidos antes de efectuar un procedimiento quirúrgico reconstructivo. ${ }^{23}$

Saavedra y colaboradores (2009), en su estudio con 63 pacientes tratados mediante uretrotomía interna en el Hospital General Dr. Manuel Gea González, entre 2000 y 2008, registraron una edad promedio de 58 años (21-86) y como causa más frecuente la iatrogénica. El promedio de Qmax preoperatorio fue de $4.1 \mathrm{~mL} / \mathrm{seg}(0-11)$ y la longitud de la estenosis de $0.6 \mathrm{~cm}(0.4-2)$, valorada por uretrocistografía; en 37 (59\%) casos la estenosis se encontró en la uretra bulbar. Al analizar la longitud del segmento estenósico en general, se observó que los pacientes con estenosis mayor de $1 \mathrm{~cm}$ tuvieron menos tiempo libre de recurrencia $(p<0.0007) .{ }^{24}$

Los datos más recientes, publicados en Estados Unidos de uretrotomía interna, reportados por Santucci y sus coautores (2010), se basan en una revisión retrospectiva de 136 pacientes (19942009), con edad media de 53 años (límites del 17 a 100) y longitud de la estenosis de $15 \mathrm{~mm}$ (2 a $50 \mathrm{~mm}$ ). La mayor parte de las estenosis se originaron en la uretra bulbar (49\%) y la tasa libre de enfermedad después de la primera uretrotomía fue de $8 \%$, con mediana de tiempo hasta la recurrencia de siete meses. ${ }^{23}$

\section{CONCLUSIONES}

Este estudio demuestra que la uretroplastia anastomósica es el tratamiento de primera línea en pacientes con estenosis uretral, específicamente en quienes sufren estenosis de localización bulbar, con una longitud igual o menor de $2 \mathrm{~cm}$. 
La uretrotomía interna representa la técnica quirúrgica de mínima invasión con mayor disponibilidad para la práctica urológica; sin embargo, tiene una alta tasa de recurrencia y no cuenta con la ventaja de la escisión del segmento uretral estenósico.

La falta de consenso del tratamiento y las diversas alternativas quirúrgicas, cuyos resultados a corto y largo plazos han sido poco satisfactorios, sugieren la necesidad de estandarizar la toma de decisiones para implementar el protocolo primario adecuado de la estenosis de uretra bulbar.

La técnica quirúrgica abierta versus endoscópica muestra menor tasa de recurrencia a seis meses. La uroflujometría es el estudio con mejor parámetro clínico para el seguimiento posquirúrgico de los pacientes con estenosis de uretra bulbar.

\section{Conflictos de Interés}

No existen.

\section{REFERENCIAS}

1. Mangera A, Chapple C. Urethral stricture disease. Surgery (Oxford) 2014;32(6):304-309.

2. Latini JM, McAninch JW, Brandes SB, et al. SIU/ICUD Consultation on urethral strictures: Epidemiology, etiology, anatomy, and nomenclature of urethral stenoses, strictures, and pelvic fracture urethral disruption injuries. Urology. 2014;83(3 Suppl):S1-7.

3. Anger JT, Santucci R, Grossberg AL, et al. The morbidity of urethral stricture disease among male medicare beneficiaries. BMC Urol. 2010;10:3.

4. Prevención, diagnóstico y tratamiento de estrechez (estenosis) de uretra postraumática por estenosis y por procedimientos terapéuticos en el hombre adulto. México: I Secretaría de Salud, 2010. Disponible en: www.cenetec. salud.gob.mx/interior/gpc.html

5. Lazzeri M, Sansalone S, Guazzoni G, et al. Incidence, Causes, and Complications of Urethral Stricture Disease. Eur Urol Suppl. 2016;15(1):2-6.

6. Fenton AS, Morey AF, Aviles R, et al. Anterior urethral strictures: Etiology and characteristics. Urology. 2005;65:1055-8.

7. Stein DM, Thum DJ, Barbagli G, et al. A geographic analysis of male urethral stricture aetiology and location. BJU Int. 2013;112:830-834
8. Palminteri E, Berdondini E, Verze $\mathrm{P}$, et al. Contemporary urethral stricture characteristics in the developed world. Urology. 2013;81:191-6.

9. Angermeier KW, Rourke KF, Dubey D, et al. SIU/ICUD consultation on urethral strictures: Evaluation and follow-up. Urology. 2014;83(3 Suppl):S8-17.

10. Gallentine ML, Morey AF. Imaging of the male urethra for stricture disease. Urol Clin North Am. 2002;29:361-72.

11. Tam CA, Voelzke BB, Elliott SP. Critical analysis of the use of uroflowmetry for urethral stricture disease surveillance. Urology. 2016;91:197-202.

12. Schafer W, Abrams P, Liao L. Good Urodynamic Practices: Uroflujometry Filling Cystometry, and Pressure-Flow Studies. Neurourol Urodyn. 2002:21:261-274.

13. Jackson MJ, Veeratterapillay R, Harding CK, et al. Intermittent self-dilatation for urethral stricture disease in males. Cochrane Database Syst Rev. 2014;12:CD010258.

14. Buckley JC, Heyns C, Gilling P, et al. SIU/ICUD consultation on urethral strictures: Dilation, internal urethrotomy, and stenting of male anterior urethral strictures. Urology. 2014;83 (3 Suppl):S18-22.

15. Morey AF, Watkin N, Shenfeld O, et al. SIU/ICUD consultation on urethral strictures: Anterior urethra-Primary anastomosis. Urology. 2014;83(3 Suppl):S23-6.

16. Terlecki RP, Steele MC, Valadez C, et al. Grafts are unnecessary for proximal bulbar reconstruction J Urol, 2010;184:2395-2399.

17. Barbagli G, Fossati N, Sansalone S, et al. Prediction of early and late complications after oral mucosal graft harvesting: multivariable analysis from a cohort of 553 consecutive patients. J Urol, 2014;191:688-693.

18. Morey AF, Watkin N, Shenfeld O, et al. SIU/ICUD consultation on urethral strictures: anterior urethra-primary anastomosis. Urology, 2014;(83):S23-S26.

19. Granieri MA, Webster GD, Peterson AC. The evolution of urethroplasty for bulbar urethral stricture disease: lessons learned from a single center experience. J Urol 2014;192(5):1468-1472.

20. Fall BY, Diallo SY, Sarr A, Thiam A. et al. Urethroplasty for male urethral strictures: Experience from a national teaching hospital in Senegal. African J Urol 2014;20:76-81.

21. Cisneros RM, Aragón A, Morales O. Estenosis uretral: etiología y tratamiento. Experiencia en el Centro Médico ISSEMYM. Rev Mex Urol 2013;73(5):229-236.

22. Bullock TL, Brandes SB. Adult anterior strictures: a national practice patterns survey of board certified urologists in the United States. J Urol 2007;177:685-690.

23. Santucci R, Eisenberg L. Urethrotomy has a much lower success rate than previously reported. J Urol 2010;183:18591862.

24. Saavedra DV, Merayo CE, Sánchez G. Recurrencia de estenosis uretral posterior a uretrotomía interna. Rev Mex Urol 2009;69(4):15. 